\title{
Pain Analysis in Patients with Pancreatic Carcinoma: Irreversible Electroporation versus Cryoablation
}

\author{
Jiannan Li, ${ }^{1}$ Shihou Sheng, ${ }^{2}$ Kai Zhang, ${ }^{1}$ and Tongjun Liu ${ }^{1}$ \\ ${ }^{1}$ Department of General Surgery, The Second Hospital of Jilin University, Changchun, Jilin, China \\ ${ }^{2}$ Department of Gastrointestinal Colorectal and Anal Surgery, China-Japan Union Hospital of Jilin University, Changchun, Jilin, China \\ Correspondence should be addressed to Tongjun Liu; tongjunliu@163.com
}

Received 18 August 2016; Revised 31 October 2016; Accepted 29 November 2016

Academic Editor: Helmut Schoellnast

Copyright (c) 2016 Jiannan Li et al. This is an open access article distributed under the Creative Commons Attribution License, which permits unrestricted use, distribution, and reproduction in any medium, provided the original work is properly cited.

\begin{abstract}
The aim of this article is to evaluate and compare the postprocedure pain in patients with pancreatic carcinoma treated with irreversible electroporation (IRE) and cryoablation (CRYO). We compared 22 patients with 22 lesions in pancreas treated with IRE and 26 patients with 27 lesions treated with cryosurgery. All the patients in the two groups were under celiac plexus block (CPB) treatment to alleviate the postprocedure pain. A numerical rating scale (VAS) consisting of 11-point scales and the $24 \mathrm{~h}$ total hydromorphone use were recorded for the analysis of the pain level in the patients who underwent these two technologies separately. Other parameters, such as the complications and the ECOG performance status, were also noted. Statistical analysis was performed by Fisher's exact test, the Chi-square test, and Student's $t$-test. All the pancreatic carcinoma patients in our study were reported to have postprocedure pain in the two groups. But there was no significant difference in the mean pain score (4.95 (IRE) versus $4.85(\mathrm{CRYO}) ; P=0.52)$ and $24 \mathrm{~h}$ total hydromorphone use ( $3.89 \mathrm{mg}$ (IRE) versus $3.97 \mathrm{mg}(\mathrm{CRYO}) ; P=0.30)$. IRE is comparable to cryotherapy in the amount of pain that patients with pancreatic carcinoma experience.
\end{abstract}

\section{Introduction}

Lots of cancer patients would experience pain during the treating procedure, and pain occurs for about $80-85 \%$ patients with unresectable pancreatic carcinoma $[1,2]$. Pain is the most prominent clinical symptom in pancreatic cancer patients and the increasing pain would significantly decrease the patients' life quality and emotion state $[3,4]$. Nowadays, percutaneous ablation therapies, such as cryosurgery and radiofrequency ablation (RFA), become more and more popular in cancer treatments among nonsurgical patients.

Irreversible electroporation (IRE) is an emerging, nonthermal percutaneous technology which takes advantage of high voltage electric field to create "nanopores" in the membrane of the target cells, thus damaging the balance inside and outside these cells which can lead to the necrosis of the cells in the end. Compared with other minimally invasive ablation techniques, IRE has its own unique advantages, including preservation of vital structures, being uninfluenced by thermal sinks, and short ablation time [5-8]. IRE has been used in the treatment of many kinds of cancer, especially pancreatic carcinoma, and proved to be a safe and an efficient ablation technology $[9,10]$.

Previous study has suggested that IRE is comparable to RFA in the amount of pain that patients with hepatocellular carcinoma would experience [11]. However, our clinical team still believe the opinion that, compared with other ablation technologies, IRE has shorter ablation time which may result in less postprocedure pain and ablation-associated complications [12]. As a result, this study was supposed to analyze the postprocedure pain among patients who underwent IRE and cryoablation for the treatment of pancreatic carcinoma, respectively.

\section{Materials and Methods}

2.1. Patients. All enrolled patients were pathologically diagnosed with pancreatic cancer and received IRE ablation or cryotherapy. There was no control group. This study was examined and approved by the Medical Ethical Review Board of a local hospital. We reviewed 48 patients with locally pancreatic carcinoma underwent IRE (22 patients) 
and cryosurgery (26 patients) from March 2014 to May 2016 in a local hospital. In the IRE group, there were 14 males and 8 females, aged from 34 to 75 years old, with a median age of 62 years. The tumor size ranged from 2.5 to $11 \mathrm{~cm}$ in the largest diameter with the mean \pm SD diameter of $(5.25 \pm$ $2.29) \mathrm{cm}$. In the Cryosurgery group, there were 18 males and 8 females, aged from 27 to 75 years old, with a median age of 57.7 years old. The mean \pm SD diameter of the tumor was $4.98 \pm 2.13 \mathrm{~cm}$, ranged from 1.6 to $12 \mathrm{~cm}$. Three patients in the IRE group and five patients in the Cryosurgery group received two ablation sessions, and all the other patients in both groups received single ablation session. In all the patients, the diagnosis was determined through ultrasound, computed tomography (CT), MRI imaging, and pathological examination. All the patients' situations were under careful evaluation by our clinical team and were considered to be unresectable.

2.2. IRE. All the patients were under general anesthesia with sevoflurane, fentanyl, and deep neuromuscular blockade and received percutaneous irreversible electroporation in a supine position. CT was utilized to guide the IRE probes to insert into the target tumors; once the $19 \mathrm{G}$ monopolar probes were in the targeted place, we performed the NanoKnife IRE generator (AngioDynamics, NY) with the use of electrocardiographic synchronization to avoid cardiac arrhythmias. Ablation was performed with the voltage of $1,500-3,000 \mathrm{kV}, 1.5-2 \mathrm{~cm}$ electrode exposure and $1.5-2 \mathrm{~cm}$ electrode spacing. The tumors' characteristics determined the number of treatment activations and the placement styles of the electrodes to achieve complete tumor ablation.

2.3. Cryosurgery. Cryosurgery was performed through percutaneous or intraoperative approaches. We used Israel Galileo cryoablation surgery system (Cryo-Hit ${ }^{\mathrm{TM}}$ ) and $1.4 \mathrm{~mm}$ cryoprobes. A variable number (one to eight) of cryoprobes determined by the diameter of tumors were inserted into the tumors with the assistance of the ultrasound. For the tumors less than $3 \mathrm{~cm}$, a single probe was feasible; when the tumors' diameter ranged from 3 to $5 \mathrm{~cm}, 2-4$ probes were necessary, whereas larger lesions required more probes. When the probe tip was $0.5 \mathrm{~cm}$ away from tumor edge, we started the double cycle of freeze/thaw procedure. The freezing process lasted $10-15 \min \left(-160^{\circ} \mathrm{C} \pm 10^{\circ} \mathrm{C}\right)$ and formed the ice ball to encompass the entire mass of the tumor with at least a " $0.5 \mathrm{~cm}$ safe border." Then the thawing process lasted $3-5 \min \left(25^{\circ} \mathrm{C} \pm 5^{\circ} \mathrm{C}\right)$. As 2 or 3 freeze/thaw cycles were finished, we removed the cryoprobes and used the thrombinsoaked Gelfoam to pack the frozen tract to control bleeding.

2.4. Neurolytic $C P B$. All the patients in the two groups were under celiac plexus block (CPB) treatment to alleviate the postprocedure pain. The needle insertion sites in which 4$5 \mathrm{~mL}, 1 \%$ lidocaine was injected were $3.0-5.0 \mathrm{~cm}$ away from the midline at the T11-L1 intervertebral disc level. With the assistance of the CT scan, a $23 \mathrm{G}$ needle inserted through the predetermined insertion site toward the intervertebral disc until the tip of the needle penetrated it. After confirmation of the needle placement, $1 \mathrm{~mL}, 10 \%$ lidocaine with $3 \mathrm{~mL}$ contrast medium was injected. We observed the spread of the contrast medium and the immediate pain relief degree of the patients. If the spread in the target area was sufficient and the pain relief satisfactory, $5-15 \mathrm{~mL}$ of $99.5 \%$ ethanol was injected through each needle $20 \mathrm{~min}$ after the lidocaine injection.

\subsection{Data Collection. Eastern Cooperative Oncology Group} (ECOG) functional status scores were collected to assess the baseline health at the time of treatment. And each patient was noted if it was necessary to use analgesics or psychotherapeutics before the IRE or cryosurgery treatment. All the patients were monitored carefully by nurses and their $24 \mathrm{~h}$ pain was reported with an 11-point numerical rating scale. Pain was assessed in three periods of time $(0-4 \mathrm{~h}, 4-$ $12 \mathrm{~h}$, and $12-24 \mathrm{~h}$ ), and the peak pain score was recorded and used in our study. The patients' postprocedure pain was also evaluated by the $24 \mathrm{~h}$ cumulative hydromorphone use from the patient-controlled analgesia pump. Complications that were associated with the treating procedure were also noted. Other data include the number of sessions in each patient, the number of electrodes used, and adjuvant therapy.

2.6. Statistical Analysis. Statistical analysis was performed using SPSS version 22.0 and any significant results were indicated by $P<0.05$. All the parameters, including patient demographics and treatment session characteristics, were analyzed by the Fisher's exact test (ECOG performance status, analgesics and/or psychotherapeutics, tumor clinical stage, and complications), the Chi-square test (sex, adjuvant therapy, and tumor location), and Student's $t$-test (age, lesion diameter, cumulative hydromorphone use, and pain score). Although some patients received two treatment sessions, each new admission was characterized with different pain score and total amount of hydromorphone.

\section{Results}

All the parameters associated with the patients' tumor characteristics, pain scale, and cumulative hydromorphone use were presented in Table 1. There was no difference in age, sex, lesion diameter, tumor location, clinical stage of the tumors, ECOG performance, receipt of adjuvant therapy, and concurrent drug therapy between the two groups.

The number of sessions each patient received may differ in the tumors characteristics, such as size and location. The larger and more sensitive tumors which invaded the important structures required more sessions. The number of patients whose tumor located in the pancreatic head which was considered as sensitive was slightly more in the Cryotherapy group (12 in IRE group versus 15 in Cryotherapy group). As a result, the number of sessions treated was slightly more in the Cryotherapy group than in the IRE group (31 sessions in 27 versus 25 sessions in 22 lesions). There was no statistical difference in the clinical stage of the pancreatic carcinoma in the two groups $(P=0.9600)$. Furthermore, there was also no significant difference in the tumors size (mean \pm SD, $5.25 \pm 2.29 \mathrm{~cm}$ in IRE group versus $4.98 \pm 2.13 \mathrm{~cm}$ in Cryotherapy group; $P=0.6922$ ) between the two groups. 
TABLE 1: Clinical characteristics, complications, drug requirements, and pain scores of patients in the two groups.

\begin{tabular}{|c|c|c|c|}
\hline Parameter & IRE & CRYO & $P^{\mathrm{a}}$ \\
\hline Number of patients & 22 & 26 & \\
\hline Number of lesions & 22 & 27 & \\
\hline Number of sessions & 25 & 31 & \\
\hline \multicolumn{4}{|l|}{ Age (years) } \\
\hline Mean \pm SD & $62.00 \pm 9.79$ & $57.69 \pm 12.86$ & $0.1738^{\mathrm{c}}$ \\
\hline \multicolumn{4}{|l|}{ Sex } \\
\hline Male & $14(63.64 \%)$ & $18(69.23 \%)$ & \multirow{2}{*}{$0.6820^{b}$} \\
\hline Female & $8(36.36 \%)$ & $8(30.77 \%)$ & \\
\hline \multicolumn{4}{|c|}{ ECOG performance status } \\
\hline 0 & $1(4.55 \%)$ & $3(11.54 \%)$ & \multirow{3}{*}{0.8510} \\
\hline 1 & $19(86.36 \%)$ & $21(80.77 \%)$ & \\
\hline 2 & $2(9.09 \%)$ & $2(7.69 \%)$ & \\
\hline \multicolumn{4}{|l|}{ Adjuvant therapy } \\
\hline Yes & $13(59.09 \%)$ & $17(65.38 \%)$ & \multirow{2}{*}{$0.7676^{\mathrm{b}}$} \\
\hline No & $9(40.91 \%)$ & $9(34.62 \%)$ & \\
\hline \multicolumn{4}{|c|}{ Concurrent drug therapy } \\
\hline \multicolumn{4}{|c|}{ Analgesics } \\
\hline Yes & $3(13.64 \%)$ & $3(11.54 \%)$ & \multirow{2}{*}{0.7676} \\
\hline No & $19(86.36 \%)$ & $23(88.46 \%)$ & \\
\hline \multicolumn{4}{|l|}{ Psychotherapeutics } \\
\hline Yes & $1(4.55 \%)$ & $4(15.37 \%)$ & \multirow{2}{*}{0.3569} \\
\hline No & $21(95.45 \%)$ & $22(84.62 \%)$ & \\
\hline \multicolumn{4}{|l|}{ Both } \\
\hline Yes & $1(4.55 \%)$ & $3(11.54 \%)$ & \multirow{2}{*}{0.6142} \\
\hline No & $21(95.45 \%)$ & $23(88.46 \%)$ & \\
\hline \multicolumn{4}{|l|}{ Lesion diameter $(\mathrm{cm})$} \\
\hline Mean \pm SD & $5.25 \pm 2.29$ & $4.98 \pm 2.13$ & $0.6922^{c}$ \\
\hline \multicolumn{4}{|l|}{ Location } \\
\hline Head & 12 & 15 & \multirow{2}{*}{$0.8267^{\mathrm{b}}$} \\
\hline Body/tail & 10 & 11 & \\
\hline \multicolumn{4}{|l|}{ Clinical stage } \\
\hline IIa & $1(4.55 \%)$ & $2(7.69 \%)$ & \multirow{4}{*}{0.9600} \\
\hline $\mathrm{IIb}$ & $4(18.18 \%)$ & $3(11.54 \%)$ & \\
\hline III & $14(63.64 \%)$ & $17(65.38 \%)$ & \\
\hline IV & $3(13.64 \%)$ & $4(15.38 \%)$ & \\
\hline \multicolumn{4}{|l|}{ Complications } \\
\hline Present & $3(13.64 \%)$ & $2(7.69 \%)$ & \multirow{2}{*}{0.6492} \\
\hline Absent & $19(86.36 \%)$ & $24(92.31 \%)$ & \\
\hline \multicolumn{4}{|c|}{ Hydromorphone provided (mg) } \\
\hline Mean \pm SD & $3.89 \pm 1.35$ & $3.97 \pm 1.60$ & $0.2995^{\mathrm{c}}$ \\
\hline \multicolumn{4}{|l|}{ Pain score } \\
\hline Mean \pm SD & $4.95 \pm 1.94$ & $4.85 \pm 2.07$ & $0.5197^{\mathrm{c}}$ \\
\hline
\end{tabular}

${ }^{\mathrm{a}} P$ values calculated by Fisher's exact test; ${ }^{\mathrm{b}} P$ values calculated by Chi-square test; and ${ }^{\mathrm{c}} P$ values calculated by Student's $t$-test.

All the patients declared pain, and the pain scores range was similar in both groups (ranged from 1 to 8 ). Four patients (18.2\%) in IRE group and seven patients $(26.9 \%)$ in cryotherapy group had greater than 6 on pain scale. There was no significant difference in pain scores between the two groups, no matter the peak main scores (mean \pm SD, $4.95 \pm$ 1.94 in IRE group versus $4.85 \pm 2.07$ in Cryotherapy group; $P=0.5197)$ nor the pain scores recorded at different times during the first $24 \mathrm{~h}$ after procedure (Figure 1). The highest pain scores were generally noted in the $4-12$ hours after 
TABLE 2: Complications and clinical characteristics of the patients in the two groups.

\begin{tabular}{|c|c|c|c|c|c|c|c|c|}
\hline Patient number & $\begin{array}{c}\text { Age } \\
\text { (years) }\end{array}$ & Sex & IRE or CRYO & Location & Number of lesions & Clinical stage & $\begin{array}{l}\text { Largest tumor } \\
\text { diameter }(\mathrm{cm})\end{array}$ & Event \\
\hline 3 & 75 & F & IRE & Head & 1 & IIB & 3.6 & Gastroparesis \\
\hline 5 & 63 & M & IRE & Body & 1 & III & 6.3 & Ascites \\
\hline 11 & 70 & M & IRE & Head & 1 & III & 2.5 & Ascites \\
\hline 23 & 54 & $\mathrm{M}$ & CRYO & Head & 1 & IV & 5.2 & Ascites \\
\hline 30 & 66 & M & CRYO & Head & 1 & IV & 4.8 & Pleural effusion \\
\hline
\end{tabular}

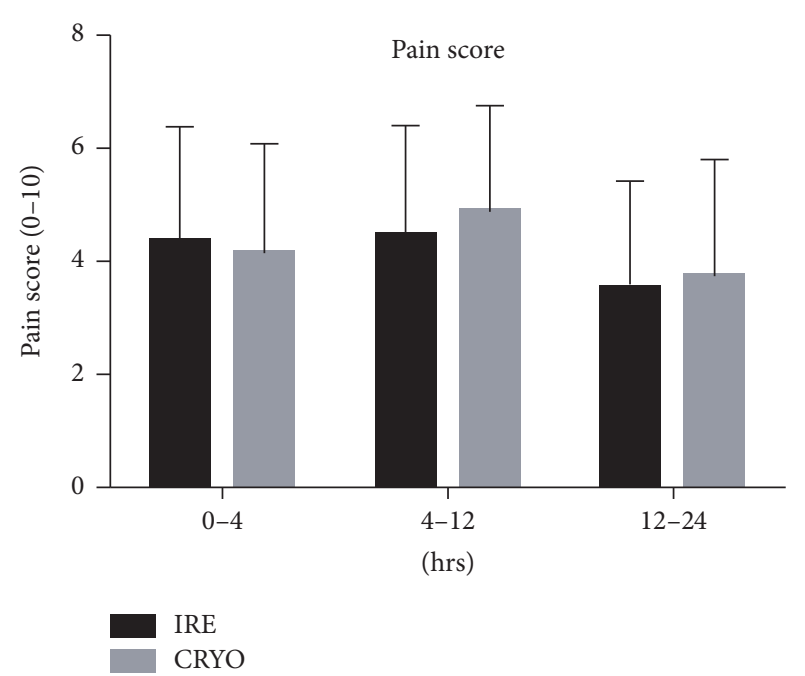

FIGURE 1: Comparison of the pain scores at different times between the two groups. There was no obvious difference of the pain scores in the two groups. The highest pain scores were noted in 4-12 hours after procedure and slightly decreased in 12-24 hours in both groups.

procedure and gradually decreased in 12-24 hours, but the extent was not so obvious (Figure 1). Additionally, there was no significant difference in $24 \mathrm{~h}$ cumulative hydromorphone use between the two groups (mean $\pm \mathrm{SD}, 3.89 \pm 1.35 \mathrm{mg}$ in IRE group versus $3.97 \pm 1.60 \mathrm{mg}$ in Cryotherapy group; $P=0.2995)$ and we also observed that the more pain patients experienced, the more hydromorphone they would like to use (Figure 2).

There was also no significant difference in the number of patients who underwent complications after procedure ( 3 in IRE group versus 2 in Cryotherapy group; $P=0.6492$ ). Complications included gastroparesis $(n=1)$ and ascites $(n=2)$ in IRE group and ascites $(n=1)$ and pleural effusion $(n=1)$ in Cryotherapy group (Table 2$)$. As for the 3 patients who had ascites after procedure, we gave them a sufficient nutrition support and growth inhibition hormone therapy, and the ascites disappeared 5-14 d after procedure. The gastroparesis patient in IRE group recovered after 2 weeks' enteral nutrition support therapy. The pleural effusion patient in Cryotherapy group was under thoracentesis and anti-infective therapy and the pleural effusion was controlled on the 6th day after cryosurgery.

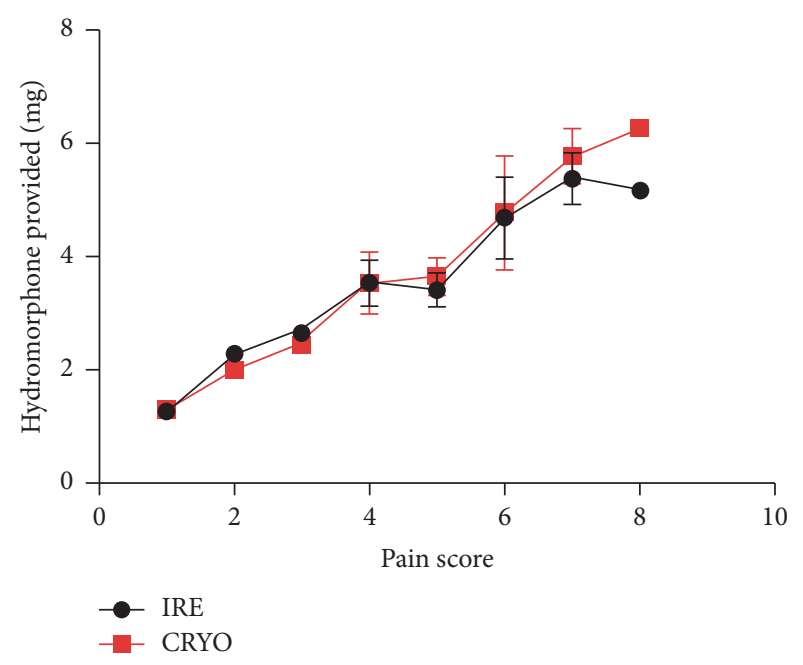

FIGURE 2: Comparison of peak pain scores with $24 \mathrm{~h}$ cumulative hydromorphone use between the two groups. Patients with more pain tended to use more hydromorphone.

\section{Discussion}

Irreversible electroporation (IRE) is an emerging and promising minimally invasive technology for the ablation of solid tumors [13-15]. It does not rely on thermal energy, but electrical field to induce cell death while preserving normal extracellular matrix, such as vessels, bile ducts, and nerves [16-18]. The preservation of vessels and extracellular matrix make it possible for the tissue regeneration in the ablated area $[19,20]$, and it may result in a fast recovery time and less pain compared with other techniques.

A pain analysis study in patients with hepatocellular carcinoma conducted by Narayanan et al. [11] compared 21 patients who underwent IRE of 29 intrahepatic lesions in 28 IRE sessions with 22 patients who underwent RFA of 27 lesions in 25 RFA sessions. They concluded that both technologies could induce low level of postprocedure pain (no pain after $46.43 \%$ of IRE sessions and $1.96 \pm 2.67$ pain scores in IRE group) and IRE is comparable to RFA in the amount of pain patients experience. Additionally, there was no difference in the cumulative use of hydromorphone within $24 \mathrm{~h}$ after procedure between the IRE and RFA groups. Another study which aimed to identify IRE-related risks and appropriate precautions for anesthetic management also 
recorded the $24 \mathrm{~h}$ postprocedure pain in 28 patients who underwent 30 IRE sessions in the liver $(n=20)$, pancreas $(n=5)$, kidney $(n=1)$, and lesser pelvis $(n=2)$ [21]. This study revealed that the postprocedure pain within $24 \mathrm{~h}$ after IRE treatment was mild in the liver, kidney, and lesser pelvis with a mean maximum reported VAS score of 3 (0-9 range). However, as for the pancreas, the pain was severe with a mean maximum VAS of 4 (2-9 range). Our findings were similar with this, with no patients declaring pain in the two groups, and the maximum VAS score (mean \pm SD) noted within $24 \mathrm{~h}$ after IRE was $(4.95 \pm 1.94)$ (range $1-8)$. Four patients in IRE group had greater than 6 on pain scale. Besides, the patients' cumulative hydromorphone use in our study in both groups was much more than that in Narayanan et al.s study [11] which was $(1.54 \pm 2.00) \mathrm{mg}$ in IRE group and $(1.24 \pm 1.19) \mathrm{mg}$ in RFA group.

Pancreatic cancer is one of the most common cancers of the digestive system, and many patients with this malignant tumor cannot be treated by complete resection due to surrounding invasion or distant metastasis. Pain is the main symptom of the patients with pancreatic cancer and severe pain would definitely decrease the quality of the patients' life. It is reported that pain in pancreatic cancer patients is more likely caused by the invasive of tumors to retroperitoneal or visceral plexus [22]. As a result, the pain scale in pancreatic cancer patients reported in our study was much more than that in the patients with hepatocellular carcinoma reported in Narayanan et al.'s study [11].

In our study, there was no significant difference in the maximum amount of pain patients experienced and the $24 \mathrm{~h}$ cumulative hydromorphone provided between the two groups. Cryosurgery is a promising percutaneous ablated therapy which has been widely used in the treatment of pancreatic cancer $[23,24]$. Thus, comparing the amount of pain induced by IRE and cryotherapy is of great importance. We also noted that there was no difference in the amount of pain recorded at different times (0-4 h, 4-12 h, and 12-24h) within $24 \mathrm{~h}$ after procedure. And the more pain patients experienced, the more total hydromorphone they would like to use.

This study was a retrospective, nonrandomized review of all patients with pancreatic carcinoma who underwent IRE and cryotherapy, and it has some limitations. We only evaluated and compared the postprocedure pain in a small number of patients in the two groups for a short time after procedure. The pain scores were collected at $0-4 \mathrm{~h}, 4-12 \mathrm{~h}$, and $12-24 \mathrm{~h}$, but the nurses did not record the pain scores at a stable time for every patient. In this case, the real peak pain scores might be missed and the recorded pain scores might be different if the patients increased the hydromorphone dosage. Additionally, we only noted the short-time postprocedure complications in both groups, but the longtime comorbidities were not recorded.

\section{Conclusions}

Our study suggests that IRE is comparable to cryotherapy in the amount of pain that patients with pancreatic carcinoma experience and there is no significant difference in the $24 \mathrm{~h}$ cumulative hydromorphone provided in the two groups. However, further randomized trials are still necessary to be performed to confirm our results.

\section{Competing Interests}

The authors declare that they have no competing interests.

\section{Acknowledgments}

The authors thank Fuda Cancer Hospital for providing the clinical data in this study. And they also thank Elixigen Corporation (Huntington Beach, California, USA) for helping in proofreading and editing the English language of final manuscript.

\section{References}

[1] L. Niu, Y. Wang, F. Yao et al., "Alleviating visceral cancer pain in patients with pancreatic cancer using cryoablation and celiac plexus block," Cryobiology, vol. 66, no. 2, pp. 105-111, 2013.

[2] J. C. Moore and D. G. Adler, "Celiac plexus neurolysis for pain relief in pancreatic cancer," Journal of Supportive Oncology, vol. 7, no. 3, pp. 83-90, 2009.

[3] I. Utne, C. Miaskowski, K. Bjordal, S. M. Paul, and T. Rustoen, "The relationships between mood disturbances and pain, hope, and quality of life in hospitalized cancer patients with pain on regularly scheduled opioid analgesic," Journal of Palliative Medicine, vol. 13, no. 3, pp. 311-318, 2010.

[4] C.-C. Lin, Y.-L. Lai, and S. E. Ward, "Effect of cancer pain on performance status, mood states, and level of hope among Taiwanese cancer patients," Journal of Pain and Symptom Management, vol. 25, no. 1, pp. 29-37, 2003.

[5] Y. J. Lee, D. S. Lu, F. Osuagwu, and C. Lassman, "Irreversible electroporation in porcine liver: short- and long-term effect on the hepatic veins and adjacent tissue by CT with pathological correlation," Investigative Radiology, vol. 47, no. 11, pp. 671-675, 2012.

[6] G. Narayanan, S. Bhatia, A. Echenique, R. Suthar, K. Barbery, and J. Yrizarry, "Vessel patency post irreversible electroporation," CardioVascular and Interventional Radiology, vol. 37, no. 6, pp. 1523-1529, 2014.

[7] S. J. Young, "Irreversible electroporation and the pancreas: what we know and where we are going?" World Journal of Gastrointestinal Surgery, vol. 7, no. 8, pp. 138-144, 2015.

[8] E. Ben-David, M. Ahmed, M. Faroja et al., "Irreversible electroporation: treatment effect is susceptible to local environment and tissue properties," Radiology, vol. 269, no. 3, pp. 738-747, 2013.

[9] M. P. Belfiore, F. M. Ronza, F. Romano et al., "Percutaneous CTguided irreversible electroporation followed by chemotherapy as a novel neoadjuvant protocol in locally advanced pancreatic cancer: our preliminary experience," International Journal of Surgery, vol. 21, no. 1, pp. S34-S39, 2015.

[10] R. C. Martin II, D. Kwon, S. Chalikonda et al., "Treatment of 200 locally advanced (Stage III) pancreatic adenocarcinoma patients with irreversible electroporation: safety and efficacy," Annals of Surgery, vol. 262, no. 3, pp. 486-492, 2015.

[11] G. Narayanan, T. Froud, K. Lo, K. J. Barbery, E. Perez-Rojas, and J. Yrizarry, "Pain analysis in patients with hepatocellular 
carcinoma: irreversible electroporation versus radiofrequency ablation-initial observations," CardioVascular and Interventional Radiology, vol. 36, no. 1, pp. 176-182, 2013.

[12] E. W. Lee, S. Thai, and S. T. Kee, "Irreversible electroporation: a novel image-guided cancer therapy," Gut and Liver, vol. 4, no. 1, pp. S99-S104, 2010.

[13] Z. Zhang, W. Li, D. Procissi, P. Tyler, R. A. Omary, and A. C. Larson, "Rapid dramatic alterations to the tumor microstructure in pancreatic cancer following irreversible electroporation ablation," Nanomedicine, vol. 9, no. 8, pp. 1181-1192, 2014.

[14] H. Scheffer, K. Nielsen, A. van Tilborg, J. M. Vieveen, M. P. van den Tol, and M. R. Meijerink, "Irreversible electroporation: a new form of image-guided tumour ablation," Nederlands Tijdschrift voor Geneeskunde, vol. 158, 2014.

[15] M. Silk, D. Tahour, G. Srimathveeravalli, S. B. Solomon, and R. H. Thornton, "The state of irreversible electroporation in interventional oncology," Seminars in Interventional Radiology, vol. 31, no. 2, pp. 111-117, 2014.

[16] Q. Luan, L. Jin, C. C. Jiang et al., "RIPK1 regulates survival of human melanoma cells upon endoplasmic reticulum stress through autophagy," Autophagy, vol. 11, no. 7, pp. 975-994, 2015.

[17] G. Narayanan, "Irreversible Electroporation," Seminars in Interventional Radiology, vol. 32, no. 4, pp. 349-355, 2015.

[18] H. J. Scheffer, K. Nielsen, M. C. De Jong et al., "Irreversible electroporation for nonthermal tumor ablation in the clinical setting: a systematic review of safety and efficacy," Journal of Vascular and Interventional Radiology, vol. 25, no. 7, pp. 9971011, 2014.

[19] G. Onik, P. Mikus, and B. Rubinsky, "Irreversible electroporation: implications for prostate ablation," Technology in Cancer Research \& Treatment, vol. 6, no. 4, pp. 295-300, 2007.

[20] Y. J. Lee, D. S. K. Lu, F. Osuagwu, and C. Lassman, "Irreversible electroporation in porcine liver: short-and long-term effect on the hepatic veins and adjacent tissue by ct with pathological correlation," Investigative Radiology, vol. 47, no. 11, pp. 671-675, 2012.

[21] K. Nielsen, H. J. Scheffer, J. M. Vieveen et al., "Anaesthetic management during open and percutaneous irreversible electroporation," British Journal of Anaesthesia, vol. 113, no. 6, pp. 985-992, 2014.

[22] D. P. Kelsen, R. Portenoy, H. Thaler, Y. Tao, and M. Brennan, "Pain as a predictor of outcome in patients with operable pancreatic carcinoma," Surgery, vol. 122, no. 1, pp. 53-59, 1997.

[23] K. Xu, L. Niu, and D. Yang, "Cryosurgery for pancreatic cancer," Gland Surgery, vol. 2, no. 1, pp. 30-39, 2013.

[24] M. G. Keane, K. Bramis, S. P. Pereira, and G. K. Fusai, "Systematic review of novel ablative methods in locally advanced pancreatic cancer," World Journal of Gastroenterology, vol. 20, no. 9, pp. 2267-2278, 2014. 


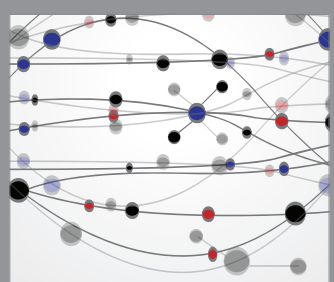

The Scientific World Journal
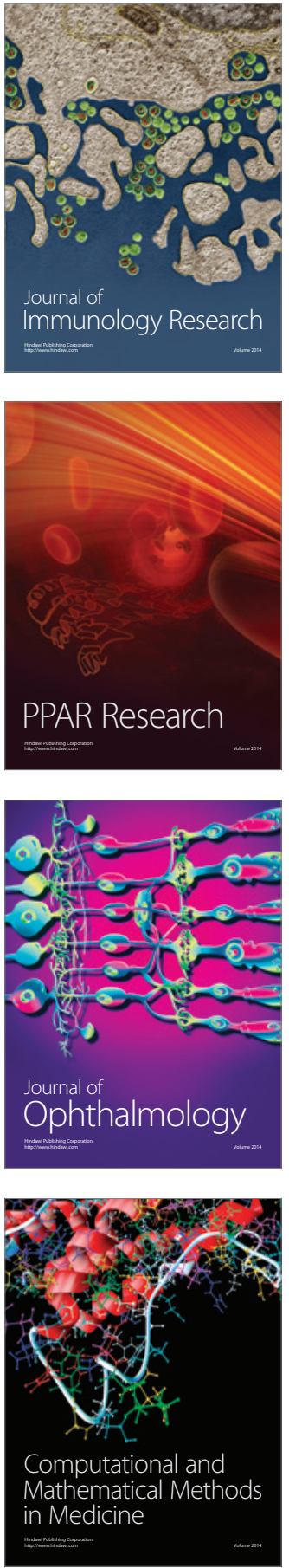

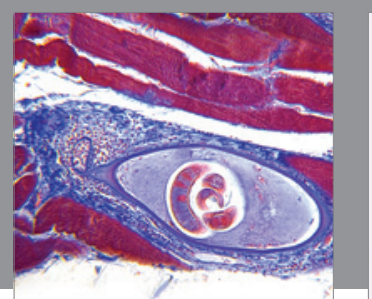

Gastroenterology Research and Practice

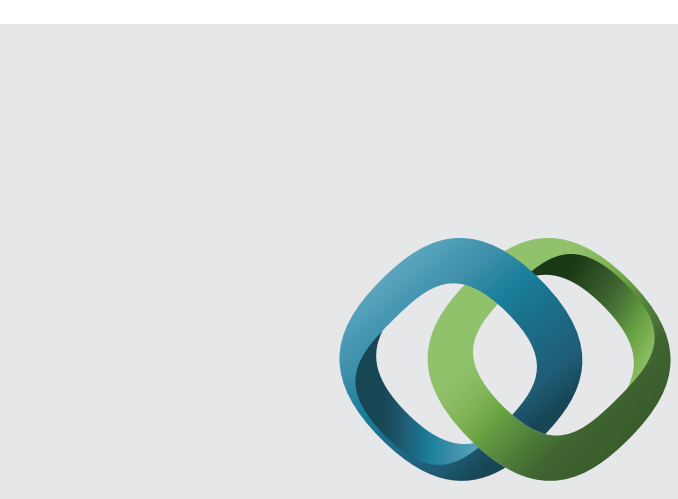

\section{Hindawi}

Submit your manuscripts at

http://www.hindawi.com
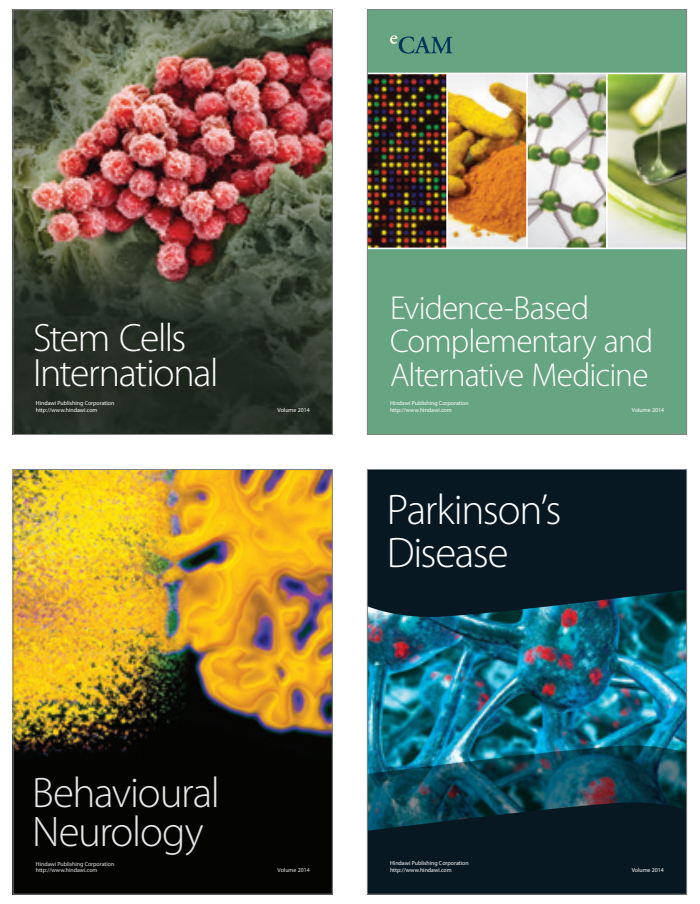
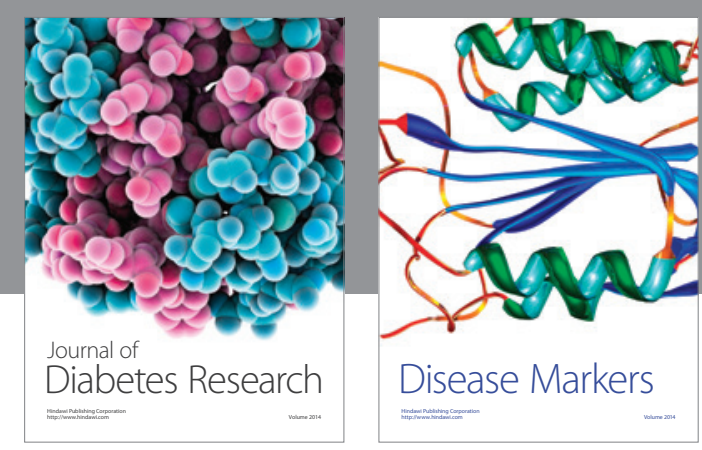

Disease Markers
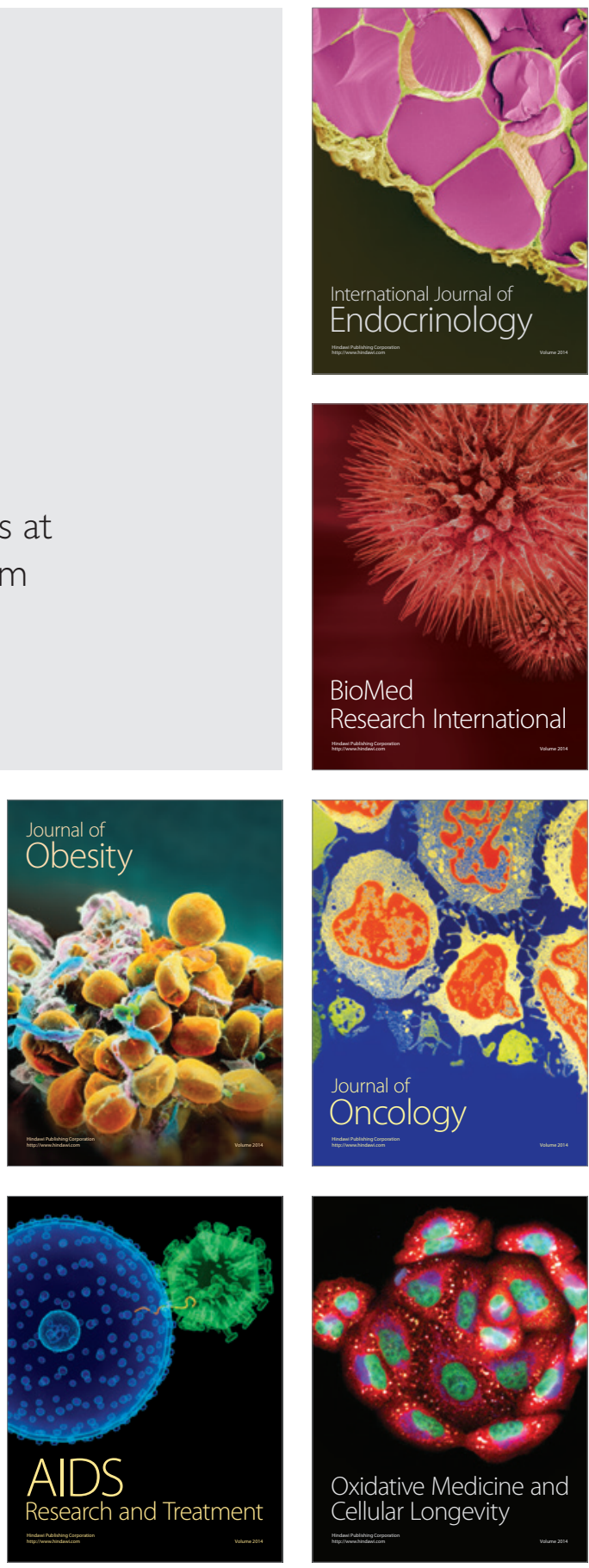